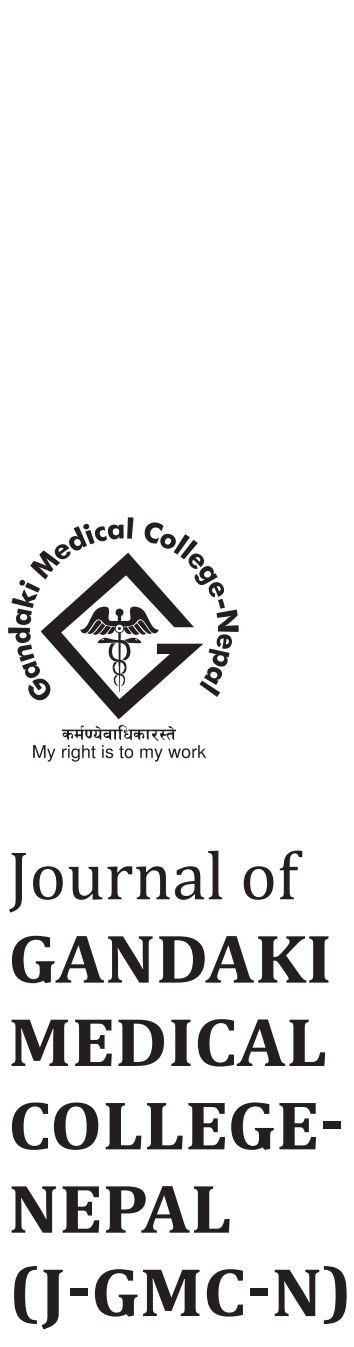

J-GMC-N | Volume $\mathbf{1 0}$ | Issue $\mathbf{0 2}$ | July-December $\mathbf{2 0 1 7}$

\section{Dengue (Break bone fever): An Emerging Disease in Nepal}

\author{
Reddy KR \\ Professor \& Head \\ Microbiology Department \\ Gandaki Medical College \& Teaching Hospital, Pokhara, Nepal
}

Dengue fever is a mosquito-borne tropical infectious disease with potential fatal complications, and highly complex pathophysiological, economic and ecologic problems, caused by dengue virus (DENV). This disease occurs primarily in the equatorial regions of Africa, the Americas, South-East Asia, and the Western Pacific. The incidence of dengue fever has increased dramatically since the 1960s, with current estimates of incidence ranging from 50 million to 528 million people infected yearly, leading to half a million hospital admissions and about 25,000 deaths. This increase is believed to be due to several factors, including global warming, urbanization, and increased international travel. Dengue has become a global problem involving newer areas, newer populations and is increasing in magnitude epidemic after epidemic, and is endemic in more than 110 countries.

Dengue fever was first referred as 'water poison' associated with flying insects in a Chinese Medical Encyclopedia from the Jin Dynasty (265 - 420 AD). The first clinically recognized dengue epidemics occurred almost simultaneously in Asia, Africa, and North America in the 1780s. The first clinical case dates from 1789 report of 1780 epidemic in Philadelphia is by physician Benjamin Rush, who coined the term 'break bone fever' because of the symptoms of myalgia and arthralgia. In the report's title he also used the term "billous remitting fever". The term dengue fever (DF) came into general use only after 1828. The word "dengue" is derived from the Swahili phrase Ka-dinga pepo, meaning "cramp like seizure".

The first epidemic of DF like illness was recorded in Madras (now Chennai) in 1780 , and the first virologically proved epidemic of DF occurred in Calcutta (now Kolkata) and Eastern Coast of India in 1963-1964. The first major epidemic of the Dengue hemorrhagic fever (DHF) occurred in Philippines followed by a quick global spread of epidemics of DF/DHF. The DHF started simmering in various parts of India since 1988. The first major wide spread epidemics of DHF and dengue shock syndrome (DSS) occurred in India in 1996 involving areas around Delhi and Lucknow and then it spread all over the country.

Although dengue virus infections have been found in India over a long period of time, there was no documented case of DF in Nepal. For the first time in 2004, dengue virus was identified as a causative agent in a patient with acute febrile illness in Nepal. Thereafter, minor dengue outbreaks were confirmed in six districts of Terai region in 2006. In addition to these, acute dengue virus infection 
has been reported from Western Nepal during 2007-2008, indicating that dengue virus infection is becoming one of the major emerging infectious disease in Nepal. It is plausible to assume that dengue virus could have been introduced into Nepal from India, due to the open border between the two countries. This hypothesis is further supported with the finding of nucleotide sequences of the Nepalese dengue strain that have been described to be very similar to the dengue virus strains circulating in India. During the 2006 outbreaks, all four dengue serotypes were found to be circulating in Nepal. In 2010, Nepal had experienced major outbreaks of dengue fever in several districts (24 districts), particularly in Chitwan and Rupendehi districts. A total of 265 cases from across the country were admitted to Sukraraj Tropical and Infectious Disease Hospital (STIDH), Kathmandu, between July and December 2010. There was one death reported due to dengue shock syndrome (DSS). Taken together, a sudden resurge of severe dengue disease can be, therefore, assumed to occur in the near future in Nepal.

Dengue virus (DENV) is a single stranded, positive-sense RNA virus of the family Flaviviridae and the genus Flavivirus. Other members of the genus include Japanese encephalitis virus, yellow fever virus, Kyasanur forest disease virus etc. Most are transmitted by arthropods (mosquitoes or ticks) and are therefore also referred to as arboviruses (ar = arthropod; $\boldsymbol{b o}=$ borne). The genome is approximately $11 \mathrm{~kb}$ in length, that encodes for three structural proteins, the capsid $(\mathrm{C})$, membrane $(\mathrm{M})$, and envelope (E) glycoproteins that form the virus particle (Fig 1), and seven nonstructural (NS) proteins (NS1, NS2A, NS2B, NS3, NS4A, NS4B, and NS5), of which NS1 has diagnostic and pathological importance. E glycoproteins are responsible for the important biological properties such as binding to receptors, hemagglutination of $\mathrm{RBC}$, and the induction of neutralizing antibodies and the protective immune response. There are four serotypes of the virus (DENV-1, DENV-2, DENV-3, and DENV-4) and recently a fifth serotype (DENV-5) has been reported in 2013.

Fig 1: Dengue virus structure (Source: en.wikipedia.org)

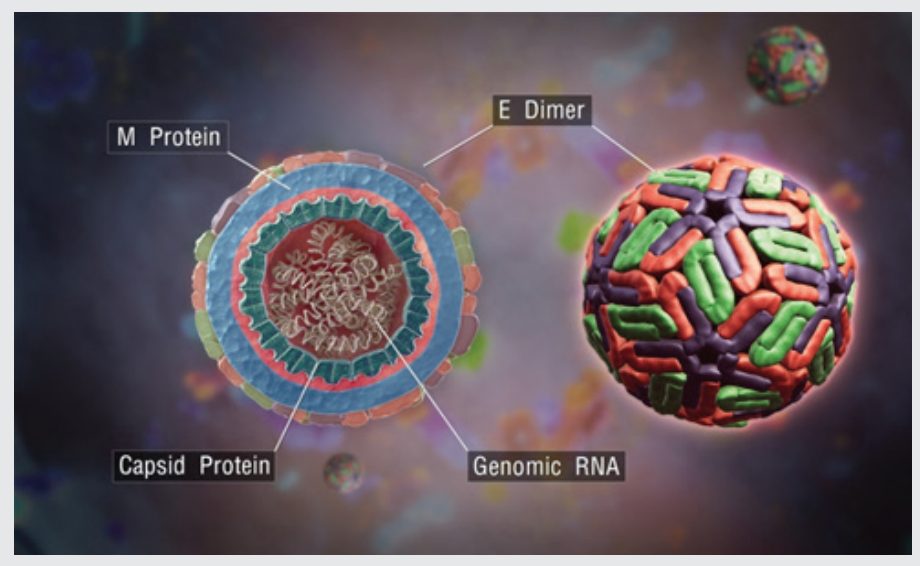

Dengue virus is transmitted primarily by domesticated day biting mosquitoes Aedes aegypti (Fig 2) and also A. albopictus. Although the mosquitoes are of Asian origin, they now occur in Africa, Europe, and USA. International travel and the transportation of goods favored the spread of both vector and virus. A female mosquito that takes a blood meal from an infected person (during the potential 2 to 12 day range of the febrile, viremic period) becomes infected (Fig 3). The virus passes from the mosquito gut to the salivary glands in 8 - 10 days (extrinsic incubation period), and is subsequently released into its saliva. The virus seems to have no detrimental effect on the mosquito, which remains infected for life. 
Fig 2: Aedes aegypti mosquito (Source: www.livemint.com)

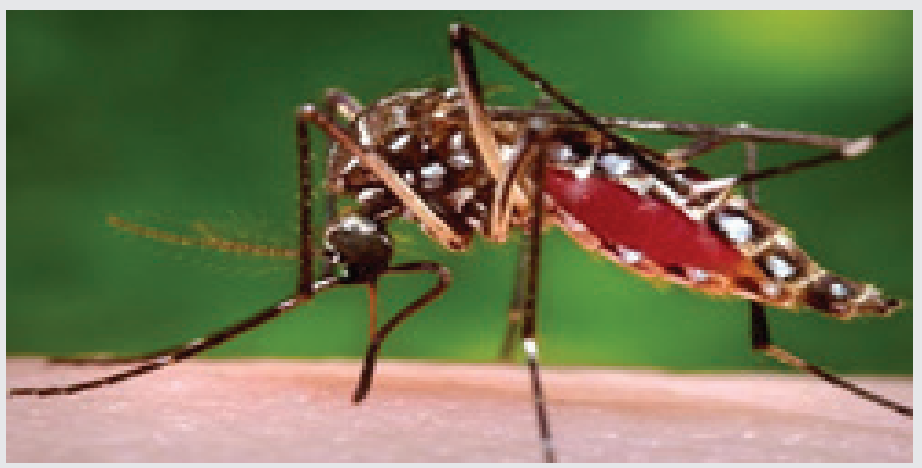

Fig 3: Transmission of dengue virus infection (Source: www.eliminatedengue.com)

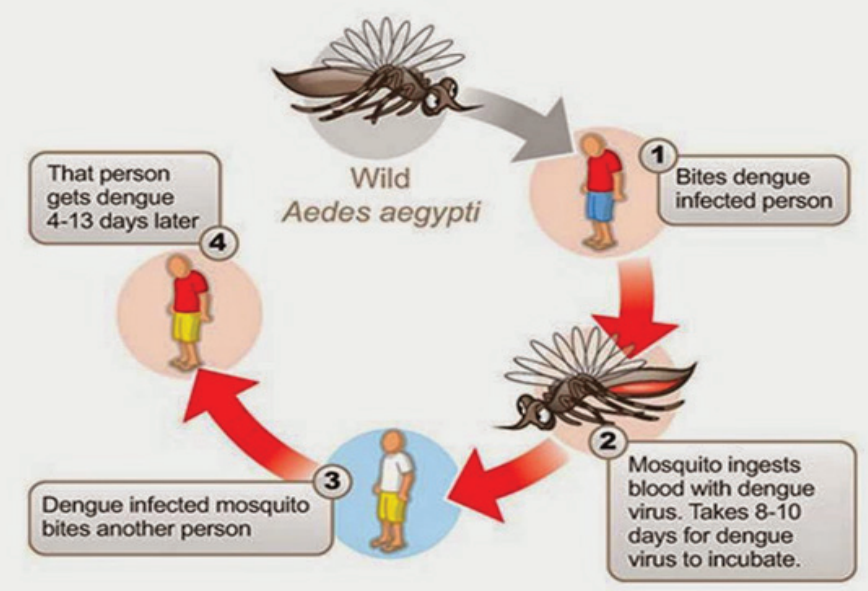

When an infected mosquito bites a person, the virus enters the skin along with the mosquito's saliva. In the skin, dengue viruses infect immature dendritic cells through the non-specific receptor (Dendritic cell-specific ICAM3-grabbing non-integrin; DC-SIGN). Infected dendritic cells mature and migrate to local or regional lymph nodes where they present viral antigens to $\mathrm{T}$ cells, initiating the cellular and humoral immune responses. There is also evidence of abundant replication of DENVs in liver parenchyma cells and in macrophages in lymph nodes, liver, and spleen, as well as in peripheral blood monocytes. Both in vitro and in vivo, macrophages and monocytes participate in antibody-dependent enhancement (ADE). This ADE occurs when mononuclear phagocytes are infected/ coated through the Fc receptors of immune complexes (Antibody-virus complexes) that form between DENVs and nonneutralizing antibodies. These nonneutralizing antibodies result from previous heterotypic DENV infection or from low concentrations of dengue antibodies of maternal origin in infant sera. The co-circulation of four DENV serotypes in a given population might be augmented by the ADE phenomenon.

Immune complexes (Antibody-virus complexes) when coated on mononuclear phagocytes by their Fc fragments of Immunoglobulins suppresses innate immune responses, increasing intracellular infection and generating inflammatory cytokines (Gamma interferon, tumor necrosis factor- $\alpha$, and interleukin-10) that lead to vascular endothelial cell dysfunction, which result in plasma leakage

DENVs produce several syndromes that are conditioned by age and immunological status. 
During initial/ primary dengue infections, most children experience subclinical infection or mild undifferentiated febrile syndromes. During secondary dengue infections, the pathophysiology of the disease changes dramatically, particularly sequential (or multiple infections with different serotypes) in which infection with DENV-1 is followed by infection with DENV-2 or DENV-3, or infection with DENV-3 is followed by infection with DENV-2. Such severe infections can result in dengue hemorrhagic fever (DHF) or dengue shock syndrome (DSS). Dengue-associated deaths are usually linked to DHF/DSS.

Infection with a given serotype confers life-long immunity to that serotype, but only shortterm immunity to others. Dengue can be life-threatening for people with chronic diseases such as diabetes mellitus and asthma.

Typically people infected with dengue virus are asymptomatic (80\%) or have only mild symptoms, such as uncomplicated fever. Others have more severe illness (5\%), and in a small proportion of cases $(<1 \%)$, it is life-threatening and causes death, despite treatment.

The incubation period ranges from 3 - 14 days, but most often it is 4 to 7 days. The onset of symptoms is usually abrupt. Fever is characteristic symptom (Febrile phase) and is often abrupt in onset with high spikes of $39.4-40.5^{\circ} \mathrm{C}$. The fever pattern is classically biphasic or saddleback, and generally lasts for five to seven days. In young children fever may cause febrile seizures or delirium. Patients with rapid defervescence may enter the critical phase of infection.

Aches and pains, particularly backache, arthralgia, myalgia, and bone pain are common. Headache is also typical of infection and is generally constant and towards the front of the head. Severe retro-orbital pain on eye movement or with a little pressure applied to the eyeball is also usual.

Gastrointestinal symptoms (e.g. anorexia, nausea or vomiting, epigastric discomfort or pain), lethargy or restlessness, collapse or dizziness may also be present. Patients often reporta lack of appetite or changes to taste sensation. Gastrointestinal symptoms, weakness, and dizziness may be more noticeable in dengue hemorrhagic fever. Upper respiratory tract symptoms, such as sore throat and cough, are usually absent.

Diffuse skin flushing of the face, neck, and chest develop early with infection. This evolves into a maculopapular or rubelliform rash of the whole body, usually on third or fourth day of the fever. Blanching may occur when the skin is pressed. The rash fades with time, and during the convalescent phase appears as pallid areas.

Hemorrhagic signs include petechiae, purpura, or a positive tourniquet test (blood pressure cuff inflated to a point midway between systolic and diastolic pressures for five minutes, and then counting any petechial hemorrhages that occur. The test is positive if $\geq 10$ petechiae per square inch appear on the forearm). More major hemorrhages can manifest as epistaxis, gingival bleeding, hematemesis, melaena, vaginal bleeding (in women of child bearing age), or bleeding from a venepuncture site. These signs can occur with either DF or DHF.

Hepatomegaly may be present. Plasma leakage is a sign of dengue hemorrhagic fever, and clinical evidence of this includes the presence of ascites, postural dizziness, or pleural effusion.

Circulatory collapse (that is, cold clammy skin, rapid and weak pulse with narrowing of pulse pressure $<20 \mathrm{mmHg}$ with decreased diastolic pressure, postural drop of blood 
pressure $>20 \mathrm{mmHg}$, capillary refill time greater than three seconds, reduced urine output) indicates the presence of shock and supports a diagnosis of DSS.

Dengue infection has three distinct phases (Fig 4): Febrile, critical, and convalescent. The febrile phase is characterized by a sudden high grade fever and dehydration that can last two to seven days. The critical phase is characterized by plasma leakage, bleeding, shock, and organ impairment and lasts for about 24 to 48 hours. It usually starts around the time of defervescence (this does not always occur), typically third to seventh day of the infection. Patients with DHF or DSS go through all three stages. The critical phase is bypassed in patients with DF.

Table 1: Main characteristic manifestations of dengue illness

1. Continuous high fever lasting $2-7$ days

2. Hemorrhagic tendency as shown by a positive tourniquet test, petechiae or epistaxis

3. Thrombocytopenia (platelet count $<100,000 \mathrm{~mm}^{-3}$ ), and

4. Evidence of plasma leakage manifested by hemoconcentration (an increase in hematocrit $20 \%$ above average for age, sex and population), pleural effusion and ascites etc.

Fig 4: Symptoms of dengue fever (Source: www.medicalnewstoday.com)

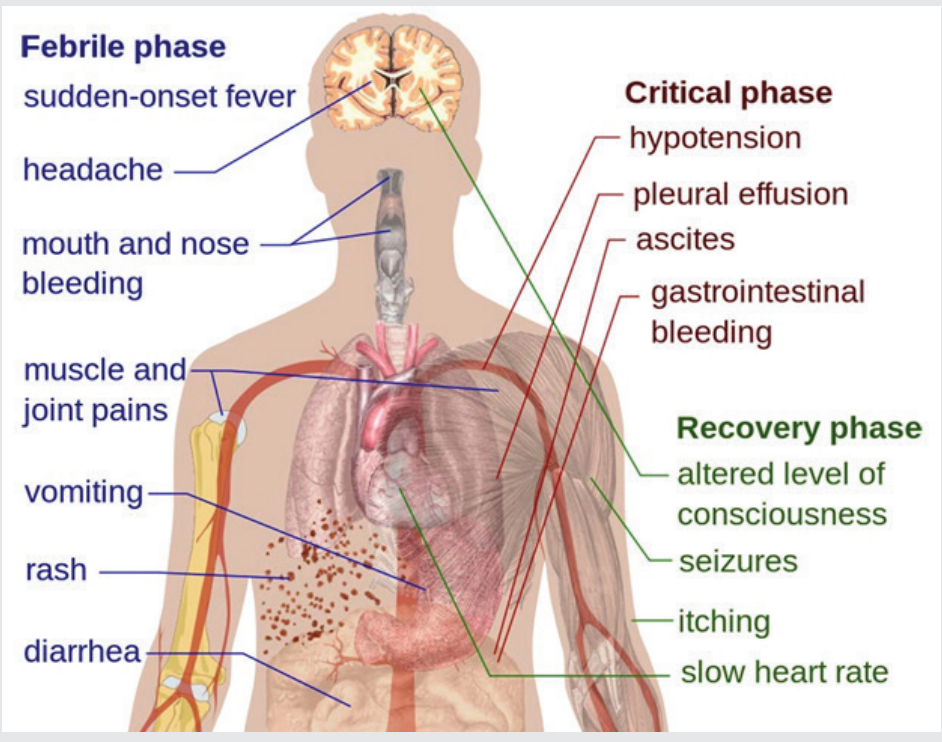

The WHO 2009 classification divides dengue fever into two groups: Uncomplicated and severe. According to this system, dengue that is associated with severe bleeding, severe organ dysfunction, or severe plasma leakage is considered severe, whereas all other cases are uncomplicated. This simplified system replaces the 1997 WHO classification, which was found to be too restrictive, although it is still widely used. The 1997 WHO classification divided dengue into undifferentiated fever, dengue fever, and dengue hemorrhagic fever (DHF). DHF was subdivided further into grades I to IV, where grade I is the presence of only easy bruising, or a positive tourniquet test result in someone with fever, grade II is the presence of spontaneous bleeding into the skin and elsewhere, grade III is clinical evidence of shock, and grade IV is shock so severe that blood pressure and pulse cannot be detected. In this system grades III and IV are referred to as 'dengue shock syndrome (DSS)'. 
The diagnosis of dengue is typically made clinically, on the basis of reported symptoms and physical examination, especially in endemic areas. A probable diagnosis is based on findings of fever and any few of the following: nausea and vomiting, rash, generalized pains, leucopenia, positive result on tourniquet test, or any warning sign (Table 2) in someone who lives in an endemic area.

Table 2: Warning signs of impending critical phase of dengue infection

- Abdominal pain or tenderness

- Persistent vomiting

- Enlargement of the liver $>2 \mathrm{~cm}$

- Mucosal bleeding

- Increase in hematocrit with rapid decrease in platelet count

- Lethargy or restlessness

- Accumulation of clinical fluid (e.g. ascites, pleural effusion)

The earliest change detectable on laboratory investigations is leucopenia, which may be followed by thrombocytopenia. Leucopenia in combination with a positive torniquet test, in a dengue endemic area has a positive predictive value of $70-80 \%$.

Table 3: Laboratory criteria for diagnosis of dengue hemorrhagic fever or dengue shock syndrome

- Rapidly developing, severe thrombocytopenia

- Decreased total WBC count and neutrophils and changing neutrophil to lymphocyte ratio

- Increased hematocrit (20\% increase from baseline is objective evidence of plasma leakage)

- Hypoalbuminemia (serum albumin $<35 \mathrm{~g} / \mathrm{L}$ suggests plasma leakage)

- Increased liver function test results (aspartate aminotransferase : alanine aminotransferase $>2$ )

The hematocrit may also rise about $10 \%$ in patients with dengue fever owing to dehydration. The results of liver function tests are usually increased, particularly for aspartate and alanine aminotransferases. Clotting studies are not required for diagnosis but may play a useful role in the management of the infection in patients with hemorrhagic signs.

Confirmatory tests should be carried out, because dengue fever can be confused with many non-dengue illnesses.

1) Detection of viral nucleic acid by PCR, and nucleic acid-sequence based amplification assay (NASBA).

2) Detection of viral antigen (NS1) in tissues such as liver, spleen, and lymph nodes as well as tissues from fatal cases (slides from paraffin-embedded, fresh or frozen tissues) by antigen-capture ELISA, and lateral flow antigen detection.

3) Serological demonstration of virus-specific antibodies (IgM, IgG) by ELISA, and 
neutralization tests.Detection of viral nucleic acid or viral antigen is primarily done in the first five days of illness, and serological tests after the fifth day.

4) Virus isolation is possible during the initial viremic phase. Aedes albopictus mosquito C6/36 cell line is the method of choice for isolation, although other mosquito (Aedes pseudoscutellaris AP61) and mammalian cell lines (Vero, LLC-MK2, BHK21 cell lines) can be used.

Imaging studies are required only if DHF or DSS is suspected. A lateral decubitus chest radiograph of the right side of the chest can be ordered to detect clinically undetectable pleural effusion in the early phase of plasma leakage.

Ultrasonography of the abdomen is useful to detect the presence of ascites and plasma leak or other disease related changes in abdominal organs, including the liver, gall bladder (edema may precede plasma leakage), and kidneys.

Treatment is supportive, as no specific antiviral therapy is available for dengue infection, and is based on guidance produced by WHO and other region specific authorities. The only recognized treatment in dengue fever is maintaining adequate hydration, and in DHF and DSS treatment is fluid replacement therapy, by judicious use of intravenous fluids to maintain sufficient urinary output and perfusion, and to achieve stabilization of vital signs, and normalization of vital signs. For patients presenting with unstable vital signs in the face of decreasing hematocrit, blood transfusion should be initiated early.

There is no vaccine available. A tetravalent vaccine is under development and may be available in the near future. Prevention thus depends on control of, and protection from the bites of, the mosquito that transmits it. The primary method of controlling Aedes aegypti is by eliminating its habitats, which include standing water in urban areas (e.g. discarded tyres, ponds, drainage ditches, and open barrels), and by applying insecticides. The mosquito bite can be avoided by appropriate clothing to cover exposed skin, especially during the day, and the use of insecticides, mosquito repellants, mosquito coils, and mosquito nets etc.

WHO is working together with several partnerships (e.g. Asia-Pacific dengue prevention partnership, European Union's DENFRAME and DENCO projects) and national Governments to develop new tools and strategies to improve diagnostics and clinical treatments and a successful vaccine. 